\title{
Editorial
}

\section{Data from Smartphones and Wearables}

\author{
Joaquín Torres-Sospedra ${ }^{1,2, *,+}$ (iD) and Aleksandr Ometov ${ }^{3, *,+}$ (iD
}

1 UBIK Geospatial Solutions S.L., 12006 Castellón, Spain

2 Institute of New Imaging Technologies, Universitat Jaume I, 12071 Castellón, Spain

3 Electrical Engineering Unit, Tampere University, 33720 Tampere, Finland

* Correspondence: jtorres@uji.es or torres@ubikgs.com (J.T.-S.); aleksandr.ometov@tuni.fi (A.O.)

+ These authors contributed equally to this work.

Citation: Torres-Sospedra, J.;

Ometov, A. Data from Smartphones and Wearables. Data 2021, 6, 45. https://doi.org/10.3390/data6050045

Received: 4 April 2021

Accepted: 24 April 2021

Published: 28 April 2021

Publisher's Note: MDPI stays neutral with regard to jurisdictional claims in published maps and institutional affiliations.

Copyright: (c) 2021 by the authors. Licensee MDPI, Basel, Switzerland. This article is an open access article distributed under the terms and conditions of the Creative Commons Attribution (CC BY) license (https:/ / creativecommons.org/licenses/by/ $4.0 /)$.
Wearables are wireless devices that we "wear" on our bodies. The proliferation of devices embedded in clothes, medical smart wear, skin patches, smartwatches, and bracelets together with conventional smartphones has broadened the collection of data from various sources for multi-disciplinary research. Nowadays, new datasets are needed for high-dimensional processing in the Big Data and Deep Learning era.

The Special Issue on "Data from Smartphones and Wearables" welcomed submissions of data descriptors, including the raw data collected by wearable devices and smartphones, as well as research articles processing this kind of data. Relevant data fields included wireless communications, tracking, indoor and outdoor positioning, eHealth monitoring, sports analysis, and gesture recognition. A total of 9 papers were published during 2020, contributing to the research community with public datasets. The published papers summed up a total of eight citations in the Web of Science (WOS) records at the moment of writing this editorial preparation.

The database described in [1], SocNav1, contains a dataset for social navigation conventions. SocNav1 aims at evaluating the robots' ability to assess the level of discomfort that their presence might generate among humans, which could be used in the future by robot navigation systems to estimate path costs. The dataset was generated using 12,500 possible scenarios, and, as a result, 12 subjects generated a total of 9280 labels for them.

A GNSS-signals database is described in [2], where raw signals were collected via roof antennas and a Spectracom simulator for general-purpose uses, including analysis for fingerprint-based applications. Radio-frequency fingerprinting has been shown to be promising for identifying transmitters and receivers, which can be used in anti-spoofing and anti-jamming solutions.

A dataset related to health monitoring of Polo ponies (heart rate and GPS location) is provided in [3]. It describes the information about the stress experienced by the Polo ponies and their cardiovascular status during the play. The provided dataset and further ones may bring additional relevant information for sport-specific nutrition, recovery strategies, tactical decisions, and play strategies.

Bluetooth and its variant Bluetooth Low Energy (BLE) play a relevant part in this Special Issue. First, a comprehensive dataset of Bluetooth Signals emitted by 27 different smartphones (six manufacturers with several models for each) is described in [4]. The signal collection and analysis were performed with a high sampling rate oscilloscope (Tektronix TDS7404), along with a low-resolution (8 bits) analog-to-digital converter. The collected data are considered useful for indoor positioning systems based on fingerprinting. Second, three independent datasets for BLE-based positioning are described in [5]. In contrast to other similar works, the authors exploited commercial beacons' features to broadcast beacon advertisements in six independent slots with different transmission parameters and analyzed their impact on the positioning accuracy. The same commercial beacons were used in [6], where BLE-GSpeed is introduced to estimate gait speed through BLE RSSI 
values. Many different devices and actors were involved in this dataset to better document a wide variety of gait speeds and walking styles.

The dataset presented in [7] was collected to improve understanding of the feasibility, usability, and validity of real-life Parkinson's disease monitoring, with a main focus on motor symptoms. To this purpose, the authors recorded objective kinematic data from accelerometers and gyroscopes in parallel as well as subjective experiential data from ecological momentary assessments. Twenty patients were monitored without daily life restrictions for fourteen consecutive days in a long-term real-life setting. The two types of data (kinematic and subjective) can be used to address hypotheses on naturalistic motor and/or non-motor symptomatology in Parkinson's disease.

Despite the above-mentioned contributions having a strong location or tracking component, other relevant datasets in other state-of-the-art fields are also relevant to the Special Issue. For instance, a dataset related to blockchain usage in smartphones is described in [8]. In particular, the database provides measurements of the impact of modern mobile blockchain projects on the battery during the turned-on display discharge process. The measurements were executed for proof-of-work and proof-of-activity consensus algorithms.

Finally, the Multilevel Monitoring of Activity and Sleep in Healthy people (MMASH) dataset is described in [9]. The database was collected by 22 participants who were monitored for $24 \mathrm{~h}$, gathering continuous psycho-physiological data, including inter-beat intervals data, heart rate data, wrist accelerometry data, sleep quality index data, physical activity, psychological characteristics, and sleep hormone levels.

To sum up, the Special Issue shows the potential of using wearable devices and smartphones as sensory devices. However, widespread donation of datasets involving wearable data is still a pending task of the research community. Having diverse datasets for the same topic may pave to way for comprehensive assessments of the proposed methods and research, not only enabling research reproducibility but also research collaborations between institutions.

Author Contributions: Both authors have equally contributed to the Special Issue management and this editorial. Both authors have read and agreed to the published version of the manuscript.

Funding: This work was supported by the European Union's Horizon 2020 Research and Innovation programme under the Marie Skłodowska Curie grant agreement No. 813278 (A-WEAR: A network for dynamic WEarable Applications with pRivacy constraints). J. Torres-Sospedra acknowledges funding from Ministerio de Ciéncia, Innovación y Universidades (Programa Torres-Quevedo, PTQ2018-009981).

Conflicts of Interest: The authors declare no conflict of interest.

\section{References}

1. Manso, L.J.; Nuñez, P.; Calderita, L.V.; Faria, D.R.; Bachiller, P. SocNav1: A Dataset to Benchmark and Learn Social Navigation Conventions. Data 2020, 5, 7. [CrossRef]

2. Morales-Ferre, R.; Wang, W.; Sanz-Abia, A.; Lohan, E.S. Identifying GNSS Signals Based on Their Radio Frequency (RF) Features-A Dataset with GNSS Raw Signals Based on Roof Antennas and Spectracom Generator. Data 2020, 5, 18. [CrossRef]

3. Best, R. Player Heart Rate Responses and Pony External Load Measures during 16-Goal Polo. Data 2020, 5, 34. [CrossRef]

4. Uzundurukan, E.; Dalveren, Y.; Kara, A. A Database for the Radio Frequency Fingerprinting of Bluetooth Devices. Data 2020, 5, 55. [CrossRef]

5. Aranda, F.J.; Parralejo, F.; Álvarez, F.J.; Torres-Sospedra, J. Multi-Slot BLE Raw Database for Accurate Positioning in Mixed Indoor/Outdoor Environments. Data 2020, 5, 67. [CrossRef]

6. Sansano-Sansano, E.; Aranda, F.J.; Montoliu, R.; Álvarez, F.J. BLE-GSpeed: A New BLE-Based Dataset to Estimate User Gait Speed. Data 2020, 5, 115. [CrossRef]

7. Habets, J.G.; Heijmans, M.; Leentjens, A.F.; Simons, C.J.; Temel, Y.; Kuijf, M.L.; Kubben, P.L.; Herff, C. A Long-Term, Real-Life Parkinson Monitoring Database Combining Unscripted Objective and Subjective Recordings. Data 2021, 6, 22. [CrossRef]

8. Bardinova, Y.; Zhidanov, K.; Bezzateev, S.; Komarov, M.; Ometov, A. Measurements of Mobile Blockchain Execution Impact on Smartphone Battery. Data 2020, 5, 66. [CrossRef]

9. Rossi, A.; Da Pozzo, E.; Menicagli, D.; Tremolanti, C.; Priami, C.; Sîrbu, A.; Clifton, D.A.; Martini, C.; Morelli, D. A Public Dataset of 24-h Multi-Levels Psycho-Physiological Responses in Young Healthy Adults. Data 2020, 5, 91. [CrossRef] 


\section{Short Biography of Authors}

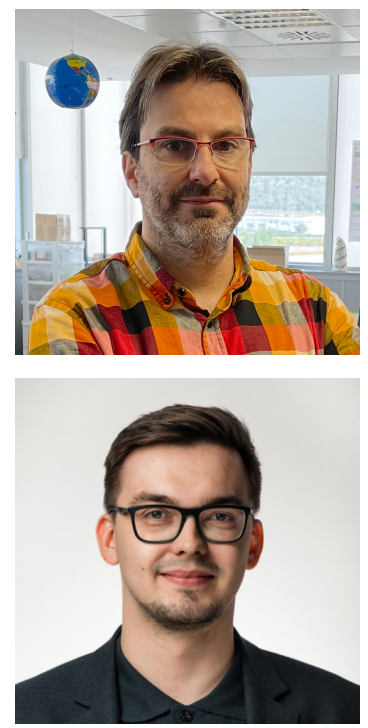

Joaquín Torres-Sospedra is CEO at UBIK Geospatial Solutions. He has a PhD since 2011 about Ensembles of Neural Networks and Machine Learning from Universitat Jaume I. He has authored more than 120 articles in journals and conference proceedings. His current research interests include indoor positioning solutions based on Wi-Fi \& BLE, Machine Learning and Evaluation. Dr. TorresSospedra is the chair of the Smartphone-based track of IPIN Competition since 2015. He is also the chair of the IPIN International Standards Committee since 2018.

Aleksandr Ometov is a Postdoctoral Research Fellow at Tampere University (TAU), Finland. He is currently working on H2020 MCSA ITN A-WEAR and APROPOS projects. He received D.Sc. (Tech) in Telecommunications and M.Sc. is Information Technology from Tampere University of Technology (TUT), Finland in 2018 and 2016, correspondingly. He also holds his Specialist degree in Information Security from Saint Petersburg State University of Aerospace Instrumentation (SUAI), Russia, 2013. His research interests are wireless communications, information security, blockchain technology and wearable applications. 